\title{
The reliability of measuring left ventricular ejection fraction by radionuclide cardiography: evaluation by the method of variance components
}

\author{
P F HØILUND-CARLSEN, * S L LAURITZEN, $\dagger$ J MARVING, * S RASMUSSEN,

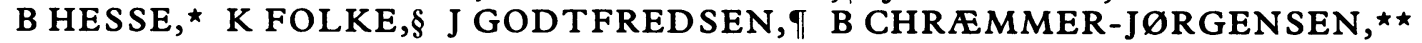 \\ N GADSBøLL, $\ddagger$ H DIGE-PETERSEN, * B HJORT JENSEN * \\ From the Departments of ${ }^{\star}$ Clinical Physiology and Nuclear Medicine, $\ddagger$ Internal Medicine $C$, Section of

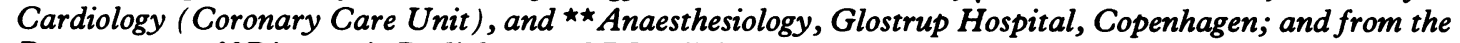 \\ Departments of §Diagnostic Radiology and $\uparrow$ Cardiology P, Gentofte Hospital, Copenhagen; and the \\ †Department of Mathematics, Statistics and Computer Science, Institute of Electronic Systems, Aalborg \\ University Centre, Denmark
}

SUMMARY A statistical model based on the method of variance components was applied to obtain confidence statements for single and repeat determinations of left ventricular ejection fraction by radionuclide techniques. With this approach variance caused by individual factors in the measurement procedure is estimated to allow calculation of confidence intervals based on single measurements and the detection limits for changes. Six study groups made up of a total of 143 subjects were examined by both multigated equilibrium and first pass imaging. Under favourable conditions (with an updated gamma camera and experienced observer) the $95 \%$ confidence interval with a single measurement of left ventricular ejection fraction by equilibrium imaging was \pm 3 ejection fraction units, compared with \pm 6 units with the first pass technique (one ejection fraction unit $=1 / 100$ of the possible values from 0.00 to 1.00 ). The minimal significant changes (at the $5 \%$ level) in measured equilibrium left ventricular ejection fraction at intervals of 15 min, 3 days, 1, 3, and 4 weeks were $\pm 4, \pm 4, \pm 5, \pm 5$, and \pm 6 units, respectively. The corresponding minimal detectable changes in a subject's "true" left ventricular ejection fraction for the same intervals were $\pm 7, \pm 7, \pm 10, \pm 10$, and \pm 12 units respectively. With first pass imaging, only average values for the variation at repeat determination could be calculated. The minimal significant change in measured first pass left ventricular ejection fraction was \pm 7 units, and the minimal detectable change in "true" left ventricular ejection fraction was \pm 14 units. Measurements of left ventricular ejection fraction by equilibrium technique were generally more reproducible than first pass determinations because the variability caused by study acquisition, observer analysis, and residual errors was smaller.

The method of variance components appears to be well suited to the evaluation of quantitative biological measurements in clinical use. The popularity of established procedures may obscure the lack of basic information about method evaluation.

Promising new quantitative laboratory methods are often evaluated by standard statistical techniques that cannot answer the questions that are most relevant to the routine clinical application of the new method. When radionuclide techniques are used to

Requests for reprints to Dr P F Høilund-Carlsen, Department of Clinical Physiology and Nuclear Medicine, Glostrup Hospital, DK-2600 Glostrup, Denmark.

Accepted for publication 9 November 1987 measure the left ventricular ejection fraction the crucial questions are: if the ejection fraction is measured once in a patient what is with $95 \%$ probability the "true" ejection fraction of the patient? And what changes in the ejection fraction can be detected at repeat determination after an hour, a day, a week, or a month? To answer these questions, we have applied a statistical approach that has rarely been used in medical studies to a comprehensive analysis of measurements of left ventricular ejection 


\section{4}

fraction by multigated radionuclide equilibrium imaging, and we have examined some aspects of the variability of measurements obtained by the gated first pass technique. The mathematical description of this method of variance components has been reported elsewhere. ${ }^{1}$ In the present paper we shall concentrate on the clinical aspects of the assessment.

\section{Patients and methods}

STUDY POPULATION

Data sets were collected from six study groups made up of a total of 143 subjects (aged 29-73 years) who gave their informed consent to the study. There were 14 healthy volunteers who were examined with approval of the Copenhagen County ScientificEthical Committee. The patients that we studied all had heart disease.

Group 1 was 29 patients with coronary artery disease examined by both equilibrium imaging and contrast ventriculography in order to determine the accuracy of the radionuclide method. Three inexperienced observers carried out the data processing of the radionuclide studies; one of them repeated the analysis later. The cineventriculograms were analysed in duplicate by an experienced radiologist.

Group 2-Twenty seven patients were randomly selected from groups 1 and 3 . In all of them one equilibrium study was followed immediately by another. The two examinations were carried out by two inexperienced observers who positioned the camera and patient and did the subsequent computer processing of the acquired data.

Group 3 was made up of 21 patients with ischaemic heart disease or chronic heart failure or both. They had two equilibrium imaging examinations 1-3 days (median 3) apart, and all data processing was performed by one inexperienced observer.

Group 4 consisted of 19 patients: 10 with previous myocardial infarction, seven with ischaemic heart disease, and two with arterial hypertension. The variation in left ventricular ejection fraction within the same hour was examined by performing four successive equilibrium studies, which were started every 15 minutes. The patients also had first pass and equilibrium imaging performed at the same time of the day at intervals of one, three, and four weeks. Data processing in this group was undertaken by an experienced observer. Data were complete for 16 of the 19 patients.

All patients in groups 3 and 4 were considered to be clinically stable and their medications were not changed during the study period.

Group 5-Four normal subjects and 30 patients with previous myocardial infarction were examined only once by equilibrium imaging. Data were proces-
Hoilund-Carlsen, Lauritzen, Marving, et al $\stackrel{\text { ㅁ }}{\stackrel{T}{\Phi}}$ sed twice by both a medium and a highly experienced observer for comparison with the results obtained by $\stackrel{\complement}{.}$ the inexperienced observers in group 1.

Group 6 consisted of 10 normal subjects and $30 \stackrel{?}{\circ}$ patients with recent myocardial infarction who were $\frac{C}{0}$ examined once by first pass and then immediately $\frac{\bar{\omega}}{\bar{n}}$. afterwards by equilibrium imaging. Data were $\mathbb{\Phi}$ processed by two highly experienced observers to compare the accuracy of first pass with that of ${ }^{\infty}$ equilibrium determinations and to measure inter and $\vec{O}$ intra observer variation with the two methods.

\section{RADIONUCLIDE CARDIOGRAPHY \\ Gated equilibrium imaging}

In all the studies we used red blood cells labelled with stannous pyrophosphate and $20-30 \mathrm{mCi}(740-1110 \%$ $\mathrm{MBq}{ }^{99 m} \mathrm{TC}$-pertechnetate. In vivo labelling was $\mathrm{W}$ used in groups 1,2 , and 3 ; and in vitro labelling in 0 group 4. We used a modified in vivo/in vitro technique $^{2}$ in groups 5 and 6 . An individual left anterior $\subseteq$ oblique view $\left(25^{\circ}-45^{\circ}\right)$ was used in all studies to give the best separation of the left ventricle from other heart chambers. In groups 1, 2, and 3, 24 frames were $\infty$ obtained per $R R$ interval in a $32 \times 32$ word matrix ${ }^{\circ}$ and a total of three million counts were collected with a single crystal gamma camera equipped with a low energy $(140 \mathrm{keV})$, high resolution, parallel hole $\overline{0}$

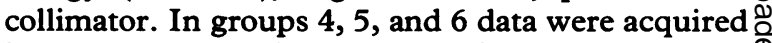
in a $64 \times 64$ word matrix and five million counts $\stackrel{\mathbb{Q}}{\mathscr{D}}$ were collected in 20 (groups 4 and 5) or 32 frames $\overrightarrow{\vec{\circ}}$ (group 6) with an updated version of the same gamma 3 camera. All data were obtained with the patients in $F$ the resting supine position. In groups 3 and 4 the angulation of the gamma camera was noted initially in each patient and reused in the repeat studies.

Under these conditions and after background correction and standardisation as $\mathbf{2 0}$ frames per $R R \frac{3}{3}$ interval for all studies, the recorded activities from the left ventricular region of interest in the end $\frac{9}{3}$ diastolic frame were on average approximately 21000 counts with in vivo labelling, 32000 counts with in vitro labelling, and 17000 with the modified $\frac{D}{0}$ in vivo/in vitro technique. These figures, however, are not directly comparable because they are influen- $\sigma$ ced by the size of the patient, the size of the left $N$ ventricle, and the proportion of high activity struc-N tures (such as the spleen) in the field of view. The ${ }_{\sigma}^{\omega}$ stability of red cell labelling was examined in the in vitro groups by comparing the concentration of activity in two blood samples drawn at an interval of $\stackrel{\mathbb{D}}{+}$ 45 minutes and counted on the collimator surface with correction for delay and radiation decay. The 0 average blood activities in the two series of samples $\overrightarrow{\mathbb{D}}$

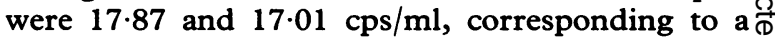
calculated biological half time of approximately $10.5 \frac{2}{2}$
hours. 
Data were initially stored on floppy discs and later analysed by a dedicated medical computer system. The ejection fraction (EF) was calculated according to the general formula:

$$
\mathrm{EF}=\frac{\mathrm{EDC}-\mathrm{ESC}}{\mathrm{EDC}},
$$

where EDC and ESC are the background corrected end diastolic and end systolic counts respectively. The outline of the left ventricle was defined by a semiautomatic edge detection algorithm. Resultant end diastolic and end systolic regions of interest were visually inspected and manually corrected whenever inaccuracies were noted. In groups 1,2 , and 3 we used a background region of interest situated inferolateral to the left ventricle in the end systolic frame, avoiding high activity structures (aorta, spleen, and gastric wall). In groups 4,5 , and 6 we used a similarly located area that contained only the pixels with lowest activity during three successive systolic frames (end systolic frame \pm one) ${ }^{3}$

\section{First pass imaging}

The first pass studies (groups 4 and 6 only) were performed as previously described ${ }^{3}$ with the gamma camera in a standard $30^{\circ}$ right anterior oblique position and before the multigated studies. In short, left ventricular ejection fraction was measured after gated reframing ( 16 frames per $R R$ interval) of the original first pass data that were collected in list mode with $\mathbf{R}$ wave trigger marks. The left ventricular outline was drawn manually on composite pictures representing end diastole and end systole respectively. For background correction we used a crescent shaped region of interest drawn along the apical border of the left ventricle on the composite end systolic picture. Left ventricular ejection fraction was calculated from the dual background corrected time-activity curves generated from the left ventricular end diastolic and end systolic regions of interest. We used the maximal activity of the diastolic region curve for end diastolic counts and the minimal activity of the systolic region curve for end systolic counts. ${ }^{3}$ With bolus injections of $20 \mathrm{mCi}(740 \mathrm{MBq})$ of ${ }^{99 \mathrm{~m}} \mathrm{Tc}$ left ventricular counts in the end diastolic frame were on average 2620 (range 860-4398) after background subtraction.

\section{Ejection fraction unit}

Left ventricular ejection fraction is a fraction, but is commonly expressed as a percentage. The term percentage is ambiguous when applied to changes in left ventricular ejection fraction. For this reason we give all confidence statements and changes in left ventricular ejection fraction in "ejection fraction units" (one unit is $1 / 100$ of the theoretical range from 0.00 to 1.00$)$.

\section{CINEVENTRICULOGRAPHY}

In group 1 single plane angiograms ( 50 frames/s) were obtained in the $30^{\circ}$ right anterior oblique projection after injection of $45 \mathrm{ml}$ of Urografin 370 (meglumine diatrizoate $(66 \%)$ and sodium diatrizoate $(10 \%)$ ) at $12 \mathrm{ml} / \mathrm{s}$. The end diastolic and end systolic outlines, including papillary muscles and trabeculations, were traced and the volumes were calculated by the area-length principle ${ }^{4}$ with a digitiser. One observer measured the left ventricular ejection fraction twice, at an interval of several months, on the original film. On each occasion the end diastolic and end systolic frames were identified by drawing the respective provisional ventricular outlines. The film was wound to and fro to ensure that these were the largest and smallest perimeters.

\section{STATISTICAL ANALYSIS}

Regression analysis per se was not part of the variance component model. It was carried out, according to the least squares principle, ${ }^{5}$ to examine the accuracy of the two radionuclide methods and to allow this to be compared with the results obtained by other methods. Possible deviation from the line of identity was tested for by an F test, ${ }^{5}$ and we used both the paired $t$ test and Pratt's non-parametric test for paired samples ${ }^{6}$ to test for differences between left ventricular ejection fraction values measured by the radionuclide and reference method.

\section{Method of variance components}

We examined the separate elements that may contribute to the total variability of the measurement of left ventricular ejection fraction. One measurement of left ventricular ejection fraction (X) was regarded as being made up of:

$$
\mathrm{X}=\xi+\Delta_{\text {time }}+\Delta_{\text {acq }}+\Delta_{\text {obs }}+\Delta_{\text {res }},
$$

where $\xi$ is the normal current level for $\mathrm{X}, \triangle_{\text {time }}$ the "error" introduced by replacing the general true value by the actual value at the time of acquisition, $\triangle_{\text {acq }}$ the error introduced by replacing the "true" value $\left(\xi+\triangle_{\text {time }}\right)$ by an examination value ( $x$ ray or radionuclide), $\triangle_{\text {obs }}$ the error caused by the observer's analysis of the acquired study, and $\triangle_{\text {res }}$ the residual error-that is the error not accounted for by any of the above. The four $\Delta s$ were assumed to be independent with a mean value $=0$ and the following variances: $\tau^{2}$ for $\Delta_{\text {time }},(\tau \sim \tau$ ime $), \alpha^{2}$ for $\triangle_{\text {acq }}(\alpha \sim$ acquisition), $\omega^{2}$ for $\triangle_{\text {obs }}(\omega \sim \omega$ bserver $)$, and $\rho^{2}$ for $\triangle_{\text {res }}(\rho \sim \rho$ esidual $)$. We have attempted to estimate the different values for these variances and their corresponding standard deviations ( $a$ ) when left ventricular ejection fraction was measured by $x$ ray or radionuclide technique; $(b)$ if the observer was experienced or less experienced; and (c) if the gamma camera was old or new. 
Results

Below we give the results of the regression analyses and the main outcome of the variance component model. All results of this model were originally calculated as variances. We give them in round figures as the more familiar standard deviations (SD), which are easier to interpret because they are expressed in ejection fraction units. It should be remembered, however, that an SD derived from several sources of error is the square root of the sum of the respective variances not the sum of the separate SDs.

\section{LEFT VENTRICULAR EJECTION FRACTION BY MULTIGATED EQUILIBRIUM IMAGING}

Accuracy.-The sets of values obtained by the two methods were not significantly different (paired $t$ test, $\rho \approx 0.64$; Pratt's test $p \gg 0 \cdot 10$ ). The regression line for cineventriculography ( $x$ axis) and equilibrium imaging (y axis) (fig 1a) was not significantly different from the opposite regression line (equilibrium technique ( $x$ axis) and cineventriculography ( $y$ axis)), and neither line differed significantly from the line of identity.

Intraobserver variation.-The variation in duplicate processing by the same observer of the same stored patient studies was larger with cineventriculography (fig 2a) than with equilibrium imaging (fig $2 b)(S D \approx 4.4$ compared with 1.9$)$. With the new gamma camera and greater observer experience the intraobserver variation at reanalysis was reduced to an SD of 1.1 for an observer of medium experience and of 1.0 for an observer of great experience.

Interobserver variation.-The variation found $c$ when the same radionuclide studies were reanalysed $\overline{\bar{s}}$ by different observers-or the "variability ofo judgment"- that is the sum of observer and residualo variances also decreased, from $S D \approx 2.5$ for an $\frac{\bar{\omega}}{1}$ inexperienced observer with the older gamma camera $\Phi$ to 1.9 for a more experienced observer and 1.1 for an very experienced observer, both with the new gamma camera.

Acquisition variation.-This was studied for only $\overrightarrow{\vec{C}}$ the older camera. Two inexperienced observers $\sigma$ performed two equilibrium studies immediately after one another in the same patients. We found an SD of approximately 0.8 , which was substantially lower ${ }^{\circ}$ than with cineventriculography. The SD foros cineventriculography was not determined separately, $\omega$ but the combined SD attributable to acquisition plus observer variation was about 6.9 . When the samegamma camera angulation was used for all studies in $\subseteq$ the same patient the acquisition SD was reduced to less than 0.3 .

Reliability.-Left ventricular ejection fraction ${ }_{\infty}^{\infty}$ measured by $x$ ray was equal to the radionuclide measurement $( \pm 1.96 \mathrm{SD})$ when the SD waso calculated from the total ( $x$ ray + radionuclide) variability of a single measurement. This composite SD was $\approx 8.6$ for an old camera and inexperienced ${ }_{\odot}^{\circ}$ observer, $\approx 8.4$ for the new camera and more $\varrho$ experienced observer, and $\approx 8 \cdot 3$ for new camera and $\overrightarrow{\vec{O}}$ a highly experienced observer. In all three instances
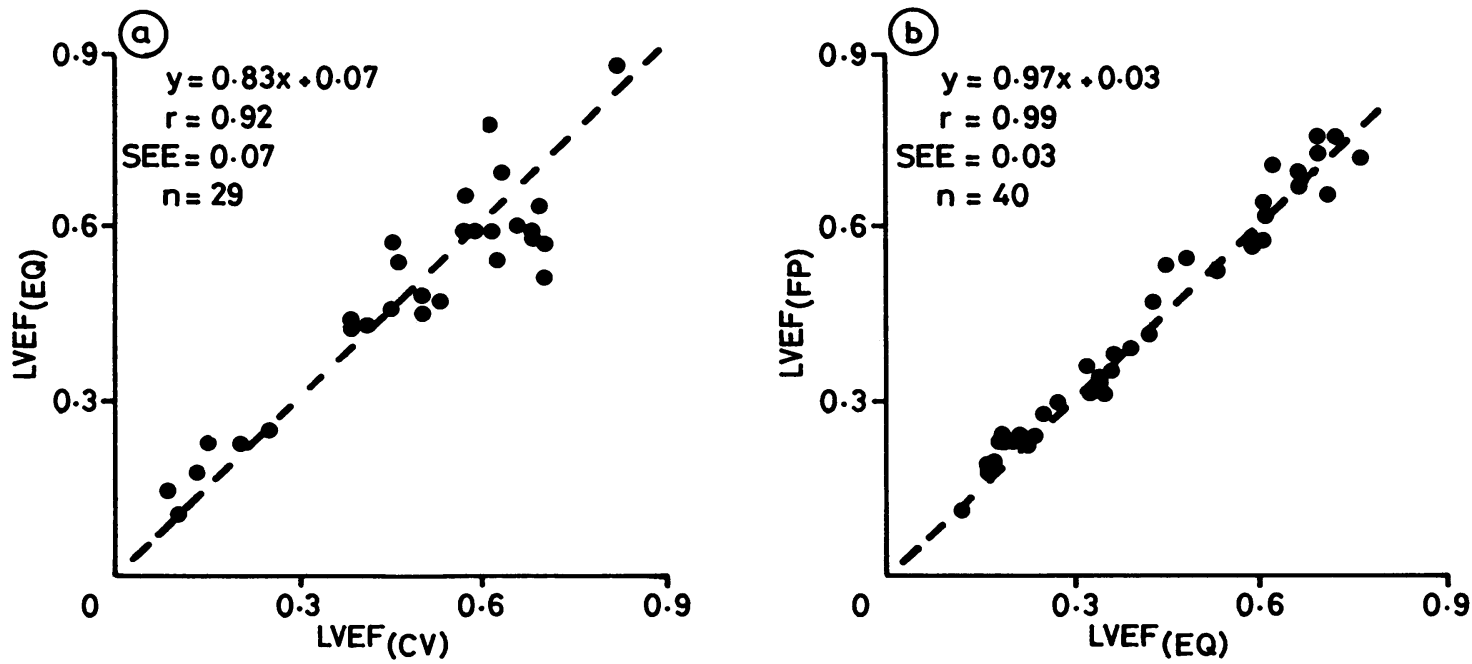

Fig 1 Relation between values for left ventricular ejection fraction ( LVEF) measured (a) by single plane cineventriculography (CV) and equilibrium imaging (EQ) and (b) by equilibrium (EQ) and first pass (FP) technique. Dotted lines indicate the line of identity. 

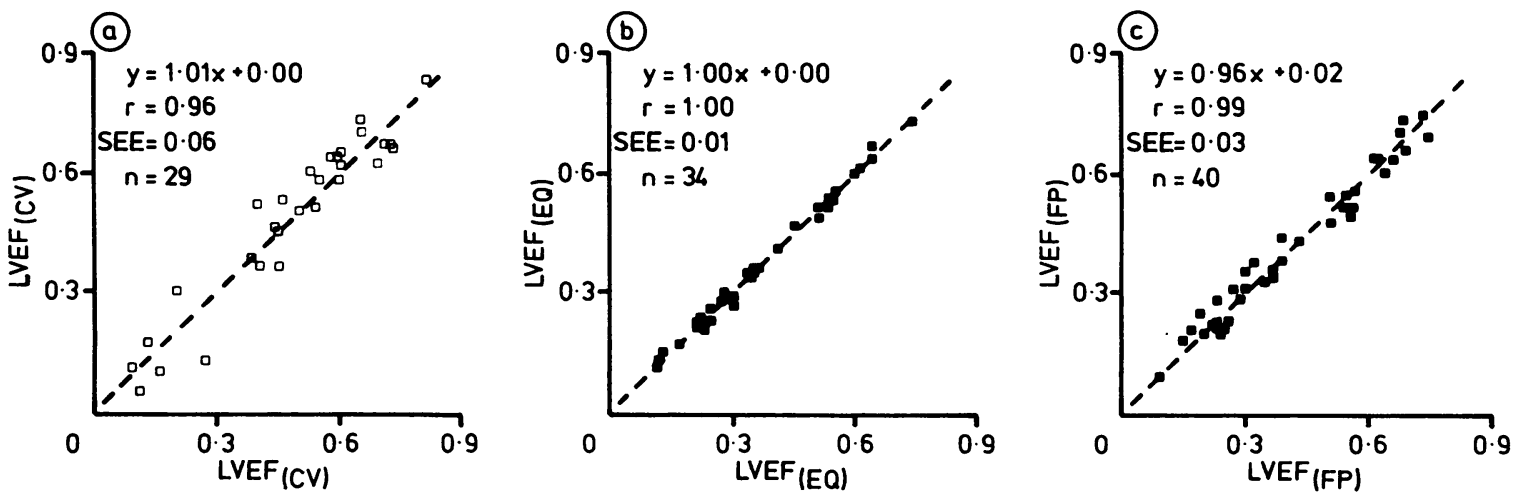

Fig 2 Intraobserver variation at recalculation of left ventricular ejection fraction ( $L V E F$ ) from patient studies obtained by (a) cineventriculography $(C V)$, (b) equilibrium imaging (EQ), and (c) first pass technique (FP). Dotted lines indicate the line of identity.
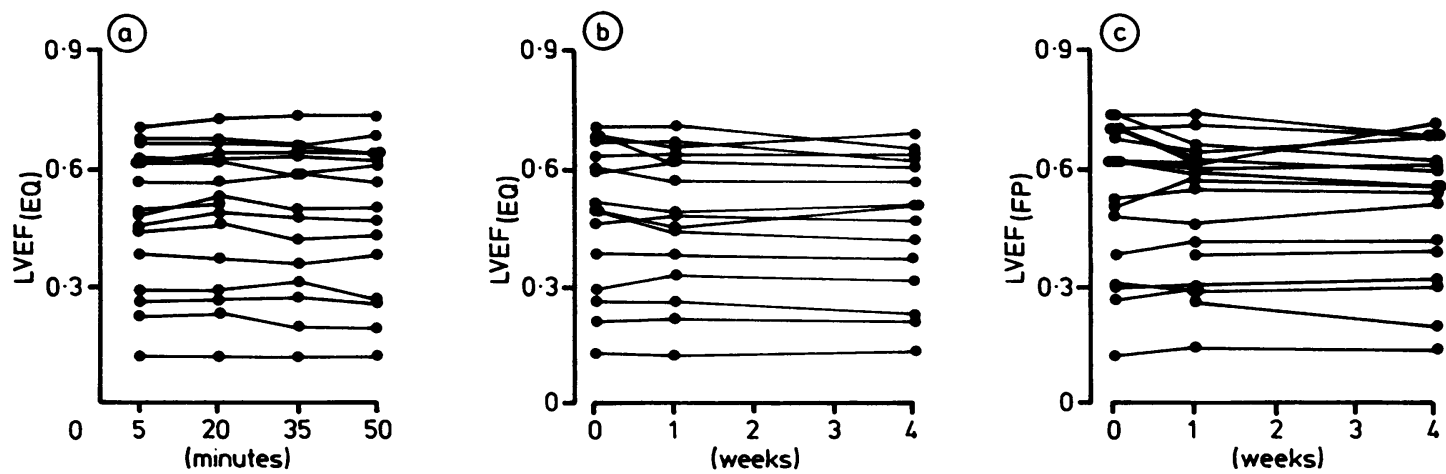

Fig 3 Variations in left ventricular ejection fraction (LVEF) for repeat measurements at different times by equilibrium imaging ( $a$ and $b$ ) and by the first pass technique (c).

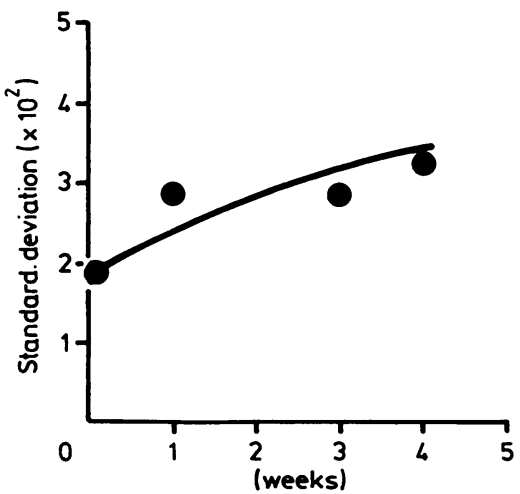

Fig 4 The standard deviation as a function of time at repeat measurement of left ventricular ejection fraction by equilibrium imaging. the SD was almost entirely attributable to radiological variation.

Variation with time.-At re-examination there were insignificant differences in heart rate and mean arterial blood pressure. Mean (SD) measured

Table 1 Estimated $95 \%$ confidence limits for single measurements of left ventricular ejection fraction (LVEF) by equilibrium $(E q)$ and first pass $\left(F_{p}\right)$ imaging

\begin{tabular}{llll}
\hline Method & Camera & $\begin{array}{l}\text { Observer } \\
\text { experience }\end{array}$ & $95 \%$ confidence interval \\
\hline Eq & Old & Low & Measured LVEF \pm 5 EF units \\
Eq & New & Medium & Measured LVEF \pm 4 EF units \\
Eq & New & High & Measured LVEF \pm EF units \\
Fp & New & High & Measured LVEF \pm 6 EF units \\
\hline
\end{tabular}

EF, ejection fraction. 
Table 2 Minimal significant changes in measured radionuclide left ventricular ejection fraction (LVEF) and minimal detectable changes in "true" LVEF (figures in parentheses) at repeat determination at different times

\begin{tabular}{|c|c|c|c|c|c|c|c|c|}
\hline \multirow{2}{*}{$\begin{array}{l}\text { Radionuclide } \\
\text { technique }\end{array}$} & \multirow{2}{*}{$\begin{array}{l}\text { Gamma } \\
\text { camera }\end{array}$} & \multirow{2}{*}{$\begin{array}{l}\text { Same } \\
\text { observer }\end{array}$} & \multirow{2}{*}{$\begin{array}{l}\text { Observer } \\
\text { experience }\end{array}$} & \multicolumn{5}{|c|}{ Interval between two studies } \\
\hline & & & & 1 hour & 3 days & 1 week & 3 weeks & 4 weeks \\
\hline $\begin{array}{l}\text { Equilibrium } \\
\text { Equilibrium } \\
\text { Equilibrium }\end{array}$ & $\begin{array}{l}\text { Old } \\
\text { New } \\
\text { New }\end{array}$ & $\begin{array}{l}\text { No } \\
\text { No } \\
\text { No }\end{array}$ & $\begin{array}{l}\text { Low } \\
\text { Medium } \\
\text { High }\end{array}$ & $\begin{array}{l} \pm 7(13) \\
\pm 6(11) \\
\pm 4(7)\end{array}$ & $\begin{array}{l} \pm 7(13) \\
\pm 6(11) \\
\pm 4(7)\end{array}$ & $\begin{array}{l} \pm 8(15) \\
\pm 7(13) \\
\pm 6(10)\end{array}$ & $\begin{array}{l} \pm 8(15) \\
\pm 7(13) \\
\pm 6(10)\end{array}$ & $\begin{array}{l} \pm 9(14) \\
\pm 8(14) \\
\pm 6(12)\end{array}$ \\
\hline $\begin{array}{l}\text { Equilibrium } \\
\text { Equilibrium } \\
\text { Equilibrium }\end{array}$ & $\begin{array}{l}\text { Old } \\
\text { New } \\
\text { New }\end{array}$ & $\begin{array}{l}\text { Yes } \\
\text { Yes } \\
\text { Yes }\end{array}$ & $\begin{array}{l}\text { Low } \\
\text { Medium } \\
\text { High }\end{array}$ & $\begin{array}{l} \pm 6(11) \\
\pm 4(7) \\
\pm 4(7)\end{array}$ & $\begin{array}{l} \pm 6(11) \\
\pm 4(7) \\
\pm 4(7)\end{array}$ & $\begin{array}{l} \pm 7(13) \\
\pm 6(11) \\
\pm 5(10)\end{array}$ & $\begin{array}{l} \pm 7(13) \\
\pm 6(11) \\
\pm 5(10)\end{array}$ & $\begin{array}{l} \pm 8(14) \\
\pm 7(12) \\
\pm 6(12)\end{array}$ \\
\hline $\begin{array}{l}\text { First pass } \\
\text { First pass }\end{array}$ & $\begin{array}{l}\text { New } \\
\text { New }\end{array}$ & $\begin{array}{l}\text { No } \\
\text { Yes }\end{array}$ & $\begin{array}{l}\text { High } \\
\text { High }\end{array}$ & & & & & $\begin{array}{l} \pm 8(15) \\
\pm 7(14)\end{array}$ \\
\hline
\end{tabular}

With first pass imaging only an average value for the variation at repeat determination could be calculated. Consequently only one set of values (placed in the 4 weeks' column) is given for this technique.

equilibrium left ventricular ejection fraction was 1 (1) (range 0-5) for reacquisition within the same hour and 3 (2) (range 0-7) after four weeks (fig 3a and b). There was a clear tendency for increasing variation with time (fig 4).

Confidence statements.-Table 1 gives the $95 \%$ confidence intervals for single measurements. Table 2 lists the values of the minimal significant changes in measured left ventricular ejection fraction and the minimal changes in "true" left ventricular ejection fraction that could be detected with $95 \%$ confidence at repeat determination at different intervals.

\section{LEFT VENTRICULAR EJECTION FRACTION BY FIRST PASS IMAGING}

For the evaluation of this technique we included only values for left ventricular ejection fraction measured by the new camera and study analysis performed by two highly experienced observers.

First pass left ventricular ejection fraction values were on an average 1 ejection fraction unit higher than equilibrium left ventricular ejection fraction (range -4 to $+9, \mathrm{p}<0.01$ ), with the regression line deviating slightly from the line of identity (fig $1 \mathrm{~b}$ ). The variability was generally higher than with

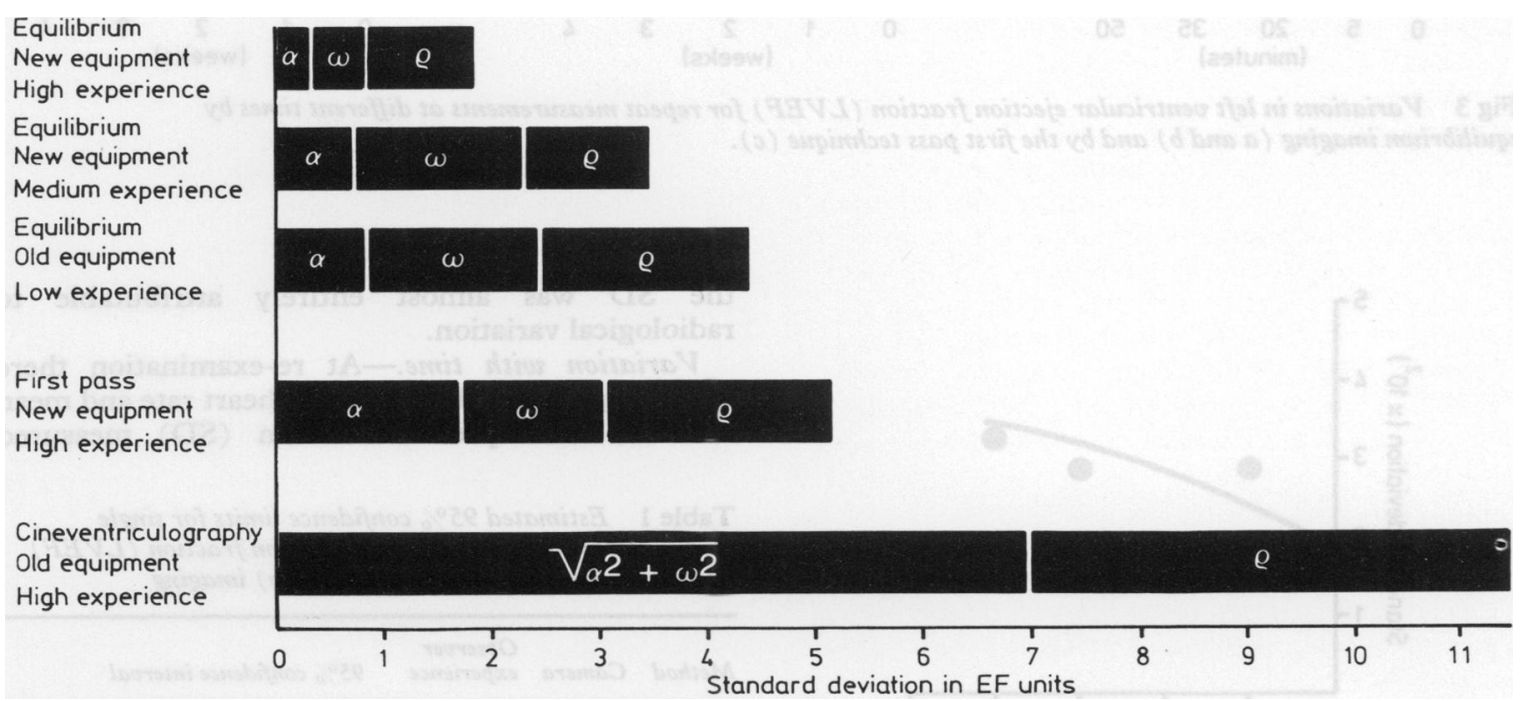

Fig 5 Graphic representation of standard deviations attributable to study acquisition ( $\alpha$ ), observer analysis (interobserver variation) ( $\omega)$, and residual errors (intraobserver analysis) ( $\rho$ ) with the three methods of measuring left ventricular ejection fraction. $\alpha$ and $\omega$ were not determined separately for cineventriculography. Standard deviations attributable to the time variation are not shown because they were calculated only for the equilibrium method. 
equilibrium imaging. Thus the standard deviations of first pass acquisition, observer, and residual variances were $1 \cdot 7,1 \cdot 3$, and $2 \cdot 1$, respectively, compared with equilibrium worst case and best case figures of $0.8,1.6,1.9$, and $0.3,0.5,1.2$ respectively, and compared with the much greater radiological variances (fig 5), in which, for example, there was an observer SD of $4 \cdot 3$. The reliability was: equilibrium left ventricular ejection fraction $=$ (first pass left ventricular ejection fraction -1$) \pm 7 \mathrm{EF}$ units.

Table 1 gives the confidence interval for single determinations of the left ventricular ejection fraction measured by the first pass technique. Table 2 gives the average detection limits at repeat measurement within four weeks.

\section{Discussion}

In method comparison studies many workers often use correlation analysis without considering whether this is a valid approach. As Altman and Bland pointed out, ${ }^{7}$ the correlation coefficient does not measure agreement; it measures association. When two methods are compared the correlation coefficient depends on the range of the true values and the measurement errors of both methods. If the range of values is wide compared with the measurement error the $r$ value will be high, whereas if the range is narrow and the methods are imprecise the correlation will be low. ${ }^{7}$

Another commonly misused technique is linear regression analysis; testing the slope of the regression line against zero is equivalent to testing the correlation coefficient. ${ }^{7}$ With the least squares principle there is a tendency for the slope to be underestimated, and in addition many ignore the error inherent in any measurement of either dependent or independent variables. To compensate for this, we carried out the regression analysis twice, first using $x$ ray left ventricular ejection fraction as the dependent variable, and then with radionuclide left ventricular ejection fraction as the dependent variable. We used regression analysis to examine the accuracy of both radionuclide methods so that our results could be compared with previously published results.

Other and more simple approaches have been proposed for method comparison studies ${ }^{7}$ because linear regression is not suitable for estimation of the relative precision of the methods compared. Instead we used a variance component model. Before discussing the application, we review other reports on the evaluation of radionuclide measurements of left ventricular ejection fraction.

PREVIOUS INVESTIGATIONS

Correlation coefficients in studies that compared first pass left ventricular ejection fraction with single plane cineventriculography range from 0.79 to 0.97 (mean 0.89$).^{8-17}$ The few reported standard errors of the estimate (SEE) range from 3 to 8 ejection fraction units (mean 6 units)..$^{811-1417}$ In studies to compare multigated equilibrium imaging and single plane ventriculography the average $r$ value was 0.84 (range $0 \cdot 70-0.92),{ }^{10121618-21}$ and values for SEE were 11,5 , and 7 units. ${ }^{121921}$ Comparisons with biplane ventriculography gave an $r$ value of 0.94 (SEE 7) with the first pass technique, ${ }^{22}$ and values of 0.88 (SEE 6 ) and 0.93 with the equilibrium method..$^{193}$

It is difficult to compare the reported results on reproducibility. The radionuclide methods differ considerably, ${ }^{24}$ and the variability of repeat assessments is influenced by the selection of patients and by the proportion of patients with normal left ventricular ejection fractions. ${ }^{16}$ Some studies are small, ${ }^{1125}$ and most deal with only a few aspects of reproducibility. ${ }^{11} 19^{2025-29}$ There is a tendency to use the same incorrect methods, and variability is expressed in various ways without taking into account all the contributory factors. This precludes the calculation of the relevant confidence intervals and detection limits. It is seldom stated how mean differences between sets of values were calculated, with sign or as the average numerical difference, or if a mean difference of $\mathrm{X} \%$ was the percentage of an ejection fraction value or the difference expressed in ejection fraction units. Even more elaborate studies $^{162}$ use conventional methods that cannot give estimates that answer the most pertinent questions.

\section{PRESENT INVESTIGATION}

Because we cannot measure true left ventricular ejection fraction we cannot be certain that a method is accurate if there is no standard with which to compare it. What we can do is to compare two methods, which we believe measure what they are intended to do, and if we find "sufficient" agreement we postulate that they are accurate. The degree of disagreement that is tolerated is determined arbitrarily.

In the present study we found no statistical differences between equilibrium imaging left ventricular ejection fraction and angiographic left ventricular ejection fraction by linear regression or by two tests for matched pairs. On the basis of these results we decided that the radionuclide method was accurate. The outcome of the regression analysis was similar to that which has been reported by others. ${ }^{1012161921}$ We assumed that the two methods measured the same variable, albeit with different precision. Because the variability of the radiological method was so much greater (fig 5) it seemed irrelevant to calculate and use for later corrections an equation that expressed the "reliability" of the 
radionuclide method-that is its ability to predict the corresponding radiological left ventricular ejection fraction. Figure 1 shows that the variability of equilibrium imaging left ventricular ejection fraction was independent of the absolute magnitude of left ventricular ejection fraction. Consequently, all variances, changes, etc, were given in absolute figures. It seemed inappropriate to express the variability of ejection fraction determinations as the coefficient of variation, because this relates the standard deviation to the mean value, which may be unrepresentative of the individual patient.

First pass values were on average one unit higher than equilibrium imaging left ventricular ejection fraction, in accordance with the finding in a previous study. ${ }^{3}$ This minor discrepancy might reflect differences between methods in background correction and delineation of the aortic valve plane.

With the variance component model we could demonstrate changes in variability when the circumstances of measurement were changed. For single determinations of left ventricular ejection fraction the $95 \%$ confidence interval fell from \pm 5 ejection fraction units with the old camera and an inexperienced observer to \pm 3 units with the new camera and an experienced observer (table 1). Because the variability of the first pass method was usually higher (fig 5) the confidence interval with new equipment and an experienced observer was wider than for equilibrium imaging with an old camera and an inexperienced observer (table 1).

The reliability of measuring changes in ejection fraction by a particular method may be judged in two ways. Both depend upon $(a)$ the inherent variability of the measurement procedure and $(b)$ what is judged to be, in advance, the acceptable frequency of false positive results, for example $5 \%$. Firstly, with repeat measurements what change in measured left ventricular ejection fraction is statistically significant (at the 5\% level)-that is, should arouse the suspicion that a real change might have taken place between two measurements? We found that the necessary change in measured left ventricular ejection fraction was $>1.96$ SD. For example, table 2 shows that when the same observer of medium experience uses the new camera for two studies at an interval of four weeks then a change of $\geqslant 7$ units in left ventricular ejection fraction is significant. Secondly, if the "true" left ventricular ejection fraction of a patient really changes between the two measurements, how large does the change need to be for it to be detected? This is partly determined by the number of false positives that we can accept. In the example chosen (table 2, 4 week column), it means that if we cannot accept more than $5 \%$, observers of medium experience will not discover a change of \pm 11 units, whereas they will probably recognise changes of \pm 12 units or more, corresponding to a change in true left ventricular ejection fraction of $\geqq$ 3.6 SD. In other words, when the true left ventricular ejection fraction changes by 12 units after four weeks, there is a $95 \%$ chance of detecting a change in measured left ventricular ejection fraction of $\geqslant 7$ units.

When the same camera position was used for repeat measurements the variability in measured left $\vec{\circ}$ ventricular ejection fraction was reduced by $<0.5$ units. With observers of medium experience variability was reduced if the same person repeated the measurement but this had little effect on the variability of measurements obtained by experienced observers (table 2).

When we used the equilibrium method variability increased with time (fig 4); this is tantamount to a decrease in the ability to detect changes in left ventricular ejection fraction (table 2). The generally higher variabilities of the first pass technique that were attributable to acquisition, observer analysis, and residual errors obscured this tendency. We calculated an average value for the total variability of 9 the first pass technique when measurements were repeated at different intervals, and we found that the average detection limits for a change in measured left ventricular ejection fraction was somewhat higher than that for comparable values with equilibrium imaging (table 2).

Our analysis was comprehensive, but not ideal statistically. It might have been improved if we had investigated some groups several times to test the sample distribution, if the data had been transformed, and if the uncertainties in estimation of the components of variance had been examined in more detail. But such procedures are often impracticable, especially when data comes from patients.

\section{CLINICAL IMPLICATIONS}

The variance component method estimates the relative impact of the many different sources of error $\frac{D}{O}$ and provides a measure of the confidence that can be put in observations. For example, cineventriculogra- $N$ phy or echocardiography in a patient with a true left $N$ ventricular ejection fraction of 0.35 -that is methods $N$ that may both have a single measurement confidence $\omega$ interval of \pm 15 ejection fraction units-may give a value from 0.20 to 0.50 . When a value lies at the extremes, the patient's global left ventricular func- $\stackrel{\Phi}{\$}$ tion could be mistakenly regarded as being either severely reduced or almost normal. The radionuclide $\overline{0}$ technique would give an equilibrium value of from $\mathbb{\Phi}$ 0.32 to 0.38 and a first pass left ventricular ejection $\frac{?}{\mathrm{D}}$ fraction of between 0.29 and 0.41 (table 1 )-that is $\varrho$ values close enough to the true value to prevent the 
functional state being put in a wrong part of the scale.

When changes in left ventricular ejection fraction are measured the need for a method with low variability is even greater. All sources of variability contribute not once, as in single measurements, but twice because the measurement is repeated, and the detection limits are not 1.96 times the composite SD but 3.60 times SD. The consequences of this are different in the clinical situation and in the scientific setting. In scientific studies a statistically significant change in left ventricular ejection fraction may be detected in a group of patients if there is a change in the same direction in a sufficiently large proportion of patients. For the clinician, however, the question is whether a measured change in left ventricular ejection fraction in an individual patient is caused by method variability or whether it reflects a real change in true left ventricular ejection fraction. If the precision of the method is not good the true answer will not emerge and the clinician will have to rely on physical signs, which, although accepted and important, are insensitive, non-specific, and not very reproducible. ${ }^{30-32}$

The evaluation of methods of measurement is lagging well behind their clinical application. Many published papers do not mention method variability. Clinicians should choose their methods of measurement after a critical appraisal of all the techniques available and not merely use the most popular or well established one.

A detailed description of the statistical model and its application to the present material can be obtained from P F H-C. This work was supported in part by the Danish Heart Foundation.

\section{References}

1 Kendall MG, Stuart A. The advanced theory of statistics. Vol 3. 3rd ed. London: Charles Griffin, 1976:58-83.

2 Callahan RJ, Froehlich JW, McKusick KA, Leppo J, Strauss HW. A modified method for the in vivo labeling of red blood cells with Tc-99m: concise communication. J Nucl Med 1982;23:315-8.

3 Marving J, Høilund-Carlsen PF, ChræmmerJørgensen B, Gadsbøll N, Hjort Jensen B. Are right and left ventricular ejection fractions equal? Ejection fractions in normal subjects and in patients with first myocardial infarction. Circulation 1985;72:502-14.

4 Dodge HT. Determination of left ventricular volume and mass. Radiol Clin North Am 1971;9:459-67.

5 Hald H. Statistical theory with engineering applications. New York and London: John Wiley, 1952:522-79.

6 Rahe AJ. Tables of critical values for the Pratt matched pair signed rank statistic. J Am Stat Assoc 1974;69: 368-73.

7 Altman DG, Bland JM. Measurements in medicine: the analysis of method comparison studies. The Statistician 1983;32:302-17.

8 Steele $P$, Kirch D, Matthews $M$, Davies $H$. Measurement of left heart ejection fraction and enddiastolic volume by a computerized, scintigraphic technique using a wedged pulmonary arterial catheter. Am J Cardiol 1974;34:179-86.

9 Steele P, LeFree M, Kirch D. Measurement of left ventricular mean circumferential fiber shortening velocity and systolic ejection rate by computerized radionuclide angiocardiography. $\mathrm{Am} J$ Cardiol 1976;37:388-93.

10 Muir AL, Hannan WJ, Brash HM, Baldwa V, Miller HC, Ogilvie B. The assessment of left ventricular ejection fraction in patients with ischaemic heart disease by contrast ventriculography and nuclear angiography. Clin Sci 1977;53:55-61.

11. Marshall RC, Berger HJ, Costin JC, et al. Assessment of cardiac performance with quantitative radionuclide angiocardiography. Sequential left ventricular ejection fraction, normalized left ventricular ejection rate, and regional wall motion. Circulation 1977; 56:820-9.

12 Folland ED, Hamilton GW, Larson SM, Kennedy JW, Williams DL, Ritchie JL. The radionuclide ejection fraction: a comparison of three radionuclide techniques with contrast angiography. $J \mathrm{Nucl} \mathrm{Med}$ 1977;18:1159-66.

13 Hecht HS, Mirell SG, Rolett EL, Blahd WH. Left ventricular ejection fraction and segmental wall motion by peripheral first-pass radionuclide angiography. J Nucl Med 1978;19:17-23.

14 Jengo JA, Mena I, Blaufuss A, Criley JM. Evaluation of left ventricular function (ejection fraction and segmental wall motion) by single pass radioisotope angiography. Circulation 1978;57:326-32.

15 Bodenheimer MM, Banka VS. Fooshee CM, Hermann GA, Helfant RH. Quantitative radionuclide angiocardiography in the right anterior oblique view: comparison with contrast ventriculography. Am J Cardiol 1978;41:718-25.

16 Wackers FJT, Berger HJ, Johnstone DE, et al. Multiple gated cardiac blood pool imaging for left ventricular ejection fraction: validation of the technique and assessment of variability. Am J Cardiol 1979;43: 1159-66.

17 Dymond DS, Halama J, Schmidt DH. Right anterior oblique first-pass radionuclide ejection fractions: effects of temporal smoothing and various background corrections. $J$ Nucl Med 1982;23:1-7.

18 Green MV, Brody WR, Douglas MA, Redwood DR, Bailey JJ, Johnston GS. Count rate measurement of left ventricular ejection fraction from gated scintigraphic images [Abstract]. $J$ Nucl Med 1976;17:557.

19 Burow RD, Strauss HW, Singleton R, et al. Analysis of left ventricular function from multiple gated acquisition cardiac blood pool imaging. Circulation 1977;56:1024-8.

20 Okada RD, Kirshenbaum HD, Kushner FG, et al. Observer variance in the qualitative evaluation of left ventricular wall motion and the quantitation of left ventricular ejection fraction using rest and exercise 
multigated blood pool imaging. Circulation 1980; 61:128-36.

21 Varani MS, Gaeta J, LeBlanc AD, et al. Validation of left ventricular volume measurements by radionuclide angiography. $J$ Nucl Med 1985;26:1394-401.

22 Shelbert HR, Verba JW, Johnson AD, et al. Nontraumatic determination of left ventricular ejection fraction by radionuclide angiocardiography. Circulation 1975;51:902-9.

23 Maddahi J, Berman D, Silverberg R, et al. Validation of two minute technique for multiple gated scintigraphic assessment of left ventricular ejection fraction and regional wall motion [Abstract]. J Nucl Med 1978; 19:669.

24 Kaul S, Boucher CA, Okada RD, Newell JB, Strauss HW, Pohost GM. Sources of variability in the radionuclide angiographic assessment of ejection fraction: a comparison of first-pass and gated equilibrium techniques. Am J Cardiol 1984;53:823-8.

25 Upton MT, Rerych SK, Newman GE, Bounous EP Jr, Jones RH. The reproducibility of radionuclide angiographic measurements of left ventricular function in normal subjects at rest and during exercise. Circulation 1980;62:126-32.

26 Marshall RC, Berger HJ, Reduto LA, Gottschalk A, Zaret BL. Variability in sequential measures of left ventricular performance assessed with radionuclide angiocardiography. Am J Cardiol 1978;41:531-6.
27 Pfisterer ME, Battler A, Swanson SM, Slutsky R, 욜 Froelicher V, Ashburn WL. Reproducibility of ejection-fraction determinations by equilibrium radionuclide angiography in response to supine $\vec{\rho}$ bicycle exercise: concise communication. J Nucl Med 1979;20:491-5.

28 Dymond DS, Elliott A, Stone D, Hendrix G, Spurrell R. Factors that affect the reproducibility of $\vec{\nabla}$ measurements of left ventricular function from first- 0 pass radionuclide ventriculograms. Circulation 1982; ڤొ 65:311-22.

29 Hecht HS, Josephson MA, Hopkins JM, Singh BN, Parzen E, Elashoff J. Reproducibility of equilibrium radionuclide ventriculography in patients with coronary artery disease: response of left ventricular ejection fraction and regional wall motion to supine bicycle exercise. Am Heart $J$ 1982;104:567-74.

30 Sloan AW, Campbell FW, Henderson AS. Incidence of the physiological third heart sound. $\mathrm{Br} M e d \mathrm{~J}$ 1952;ii:853-5.

31 Stage P, Movild B, Hesse B, Steinmetz E. Pulmonary congestion in chronic heart disease. Pulmonary, clinical and hemodynamic relationships. Acta Radiol [Diagn] (Stockh) 1976;17:417-24.

32 Eisenberg PR, Jaffe AS, Schuster DP. Clinical evaluation compared to pulmonary artery catheteriza- $\infty$ tion in the hemodynamic assessment of critically ill patients. Crit Care Med 1984;12:549-53. 